\title{
Stylistic variation among mobile speakers: Using old and new regional variables to construct complex place identity-Corrigendum
}

\author{
JENNIFER NYCZ \\ doi:10.1017/S0954394518000108, Published by Cambridge University Press, 22 October 2018.
}

Table 5 did not display properly in the above publication (Nycz, 2018). The table is correctly displayed below.

TABLE 5. Mixed-effects regression analysis of (ayT) F1 containing nation:stance interaction $\mathrm{n}=1093$

Intercept $=736.65$

\begin{tabular}{lrrrrr}
\hline \hline Factor & Coefficient & \multicolumn{1}{l}{ SE } & $\mathrm{t}$ & Tokens & Mean \\
\hline Following manner & & & & & \\
$\quad$ Fricative (baseline) & .67 & 14.57 & .05 & 151 & 780.84 \\
$\quad$ Stop & .51 & .05 & 10.47 & 942 & 747.42 \\
Duration, msec & & & & & \\
Speaker (sum contrasts) & & & & & \\
$\quad$ Victoria & -39.14 & 6.22 & -6.30 & 131 & 704.90 \\
Laurie & -34.64 & 4.67 & -7.42 & 291 & 720.51 \\
Sophie & 3.95 & 7.25 & 0.55 & 92 & 782.12 \\
Vanessa & 9.45 & 5.78 & 1.63 & 167 & 778.02 \\
Edward & 11.35 & 6.38 & 1.78 & 137 & 756.82 \\
Bob & 23.38 & 7.33 & 3.19 & 82 & 774.55 \\
Jenny & 25.64 & 5.40 & 4.75 & 193 & 781.77 \\
Nation & & & & & \\
Canada (baseline) & & & & 318 & 754.69 \\
US & -20.25 & 13.93 & -1.45 & 775 & 750.94 \\
Stance & & & & & \\
Neutral (baseline) & & & & 151 & 750.71 \\
Negative & -29.54 & 17.16 & -1.72 & 180 & 750.47 \\
Non-aligned & -25.20 & 16.35 & -1.54 & 195 & 749.42 \\
Ambivalent & -24.16 & 20.22 & -1.19 & 159 & 741.01 \\
Positive & -19.69 & 18.73 & -1.05 & 139 & 758.96 \\
$\quad$ Aligned & -15.15 & 13.98 & -1.08 & 269 & 758.65 \\
Nation:stance & 16.00 & 20.85 & 0.77 & 111 & 757.41 \\
$\quad$ US:positive & 19.80 & 22.23 & 0.89 & 138 & 741.47 \\
US:ambivalent & 24.17 & 16.71 & 1.45 & 118 & 751.81 \\
US:aligned & 37.70 & 18.62 & 2.02 & 151 & 755.41 \\
US:nonaligned & 40.45 & 19.31 & 2.10 & 141 & 751.52 \\
US:negative & & & & & \\
\hline \hline
\end{tabular}

REFERENCE

Nycz, Jennifer. (2018). Stylistic variation among mobile speakers: Using old and new regional variables to construct complex place identity. Language Variation and Change 30:175-202. doi:10.1017/ S0954394518000108 